\title{
3DMOVER 2.0 - LOW-COST APPLICATION FOR USABILITY TESTING OF 3D GEOVISUALISATIONS
}

\author{
L. Herman ${ }^{1}$ * \\ ${ }^{1}$ Department of Geography, Faculty of Science, Masaryk University, Kotlářská 2, 61137 Brno, The Czech Republic \\ herman.lu@mail.muni.cz
}

\section{Commission II}

KEYWORDS: 3D geovisualisation, 3DmoveR, usability, user logging, user testing.

\begin{abstract}
:
Three-dimensional (3D) visualisations of geospatial data have become very popular in the last years. Various applications and tools are based on interactive 3D geovisualisations. However, the user aspects of these 3D geovisualisations are not yet fully understood. While several studies have focused on how users work with these 3D geovisualisations, only few studies focus directly on interactive 3D geovisualisations and employ usability research methods like screen logging. This method enables the objective recording of movement in 3D virtual environments and of user interactions in general. Therefore, we created a web-based research tool: a 3D Movement and Interaction Recorder (3DmoveR). This tool is based on the user logging method, combined with a digital questionnaire and practical spatial tasks. The design and implementation of this tool follow the spiral model, and its current version is 2.0. It is implemented using open web technologies such as PHP, JavaScript, and the Three.js library. After building this tool, we verified it through load testing and a simple pilot test verifying accessibility. We continued to describe the first deployment of 3DmoveR 2.0 in a real user study. The future modifications and applications of 3DmoveR 2.0 are discussed in the conclusion section. Attention was paid to future deployment during user testing outside controlled (laboratory) conditions.
\end{abstract}

\section{INTRODUCTION}

The three-dimensional (3D) visualisation of geospatial data is employed today in many fields and in relation to many specific issues. Some universal applications, such as Google Earth, Cesium, or Virtual Earth, and many domain-specific solutions can be applied in various areas (Biljecki et al., 2015). However, despite the wide dissemination of 3D visualisation technologies, relatively little is known about their user aspects, usability, and theoretical background in general. For these reasons, it is important to perform user testing of 3D geovisualisations and to focus directly on the usability of interactive 3D geovisualisations because interactive $3 \mathrm{D}$ geovisualisation is the basis of the above-mentioned applications. The necessity of user evaluation, user issues, and usability, in general, can be grounded in legislative demands as Reznik (2013) shows by the example of the INSPIRE directive.

The main objective of this paper is to describe the design, implementation, evaluation, and first real application of an experimental tool for the usability testing of interactive 3D geovisualisations. This tool is called the 3D Movement and Interaction Recorder (3DmoveR) and is currently in version 2.0.

\section{RELATED WORK}

\subsection{User testing of 3D geovisualisations}

Most recent user studies employ only static 3D geovisualisations as stimuli (Schobesberger and Patterson, 2007; Engel et al., 2013; Niedomysl et al., 2013; Popelka and Brychtova, 2013; Seipel, 2013; Preppernau and Jenny, 2015; Rautenbach et al., 2016; Zhou et al., 2016; Liu et al., 2017).
However, the results of such studies cannot be transferred to interactive applications.

Some studies that have included interactive stimuli are problematic for various methodological reasons and can be mentioned here, too. Bleisch, et al. (2008) compared static 2D visualisations and interactive $3 \mathrm{D}$ ones; interaction in a $3 \mathrm{D}$ environment was enabled, but it was not monitored. Herbert and Chen (2015) tried to identify whether users preferred 2D maps and plans or interactive 3D geovisualisations in matters of spatial planning. In both these studies, two independent variables were not distinguished as separate (the level of interactivity and dimensionality of visualisation), and, hence, it was not possible to identify their true effects. Wilkening and Fabrikant (2013) studied user interaction with Google Earth, but the user strategies were only observed and manually recorded. Sprinarova et al. (2015) also described a mainly qualitative (and subjective) user study, in which participants were observed and their movement strategies in a 3D virtual environments, including a terrain models, were analysed.

\subsection{Methods of user testing}

As follows from the above, there are many approaches to evaluating geovisualisations. Thus, it is possible to use a variety of evaluation methods to derive the qualitative or quantitative characteristics of the tested visualisations.

Authors such as Van Elzakker (2004) or Li et al. (2010) provide an overview of usability methods. These are:

- questionnaires,

- interviews,

\footnotetext{
* Corresponding author
} 
- $\quad$ direct observation,

- think-aloud protocol,

- focus groups,

- $\quad$ eye-tracking,

- $\quad$ screen capture and screen logging.

All these methods are associated with solving practical tasks with the help of a tested product or subjective evaluation of the evaluated product. In the case of solving practical tasks, usually speed and accuracy of user responses is recorded and analysed. The mentioned methods are often not used individually but combined to meet the needs of the specific study. This approach is called mixed research design, which was introduced into several disciplines by Cameron (2009) and into geospatial data visualisation by Bleisch (2011) or Van Elzakker and Griffin (2013).

\subsection{Principles of user logging}

User logging is a research method that is able to record objectively and then store different usability parameters (efficiency, effectiveness, and satisfaction) when working with interactive stimuli. User logging also allows the recording of various aspects of individual user strategies. Interaction using the mouse (mouse logging) and keyboard, as well as other control devices can be recorded. This method is principally used to evaluate interactive applications and websites.

In the field of spatial data visualisation, this approach was applied, for example, by Nivala et al. (2008), when evaluating four web map portals; the aim was to identify problems in controlling these portals. The screen logging method works in a similar way, but, unlike user logging, it provides mainly qualitative data. Screen recording and user logging to improve the user-friendliness of map applications has also been employed by Pucher and Schobesberger (2011).

In practice, user and screen logging are often used in combination with an approach called $\mathrm{A} / \mathrm{B}$ testing. Users are randomly assigned one of two product variants (A or B), and, subsequently, the effect of the variant on their decision is documented. These variants of the product are created with respect to predetermined hypotheses (Speicher et al., 2014).

\subsection{Application of user logging in 3D geovisualisations testing}

As mentioned above, many of the usability studies of 3D geovisualisations dealt only with static 3D stimuli (perspective views). There are only a few studies that apply user (or screen) logging to the user evaluation of interactive 3D geovisualisations. For example, Abend et al. (2012) analysed interactive movement by screen logging; their work processed videos captured while a user worked with Google Earth. Subsequent analysis of these videos is more time demanding than evaluating screen logging data, which can be analysed automatically.

User logging had been used, for example, by Treves et al. (2015), who tracked and analysed the movement of participants in a virtual environment. Also McKenzie and Klippel (2016) examined virtual movement speed and the problem of wayfinding in a virtual environment. Jurik et al. (2017) studied interaction in interactive 3D spatial data visualisation as one part of their study. The proportion of individual movement types was recorded in interactive tasks.
As previously mentioned, most usability studies in cartography concern only static 3D geovisualisations as stimuli. If interactive movement in the $3 \mathrm{D}$ environment was possible, it was neither monitored nor analysed in detail. The studies by Wilkening and Fabrikant (2013), Treves et al. (2015), McKenzie and Klippel (2016), and Jurik et al. (2017) are the only exceptions. At the same time, it is necessary to improve upon these approaches (eliminate manual recording and support different variants of 3D geovisualisations) and combine them to allow comprehensive analysis of user interactions. Hence, we designed and implemented a new testing tool for the following reasons: to allow speed, accuracy of responses, and the subjective opinions of participants to be recorded in a mixed research design.

\section{DEVELOPMENT OF 3DMOVER}

3Dmove R (3D Movement and Interaction Recorder) is a tool that has been designed and implemented with regard to the above-listed findings. The design and implementation of 3DmoveR followed the so-called spiral model. The first version (3DmoveR 1.0) was developed after two iterations. In the first, we designed and implemented an initial prototype, which was then pilot tested. After improving the prototype based on the pilot test, we created a second version for use in another round of pilot testing. The core of 3DmoveR 1.0 was X3DOM, a JavaScript library for visualising 3D data. Other open web technologies (jQuery, PHP - Hypertext Preprocessor) were also used for its implementation. 3DmoveR 1.0 and two derived tools, 3D Touch Interaction Recorder (3DtouchR) and 3D Gaze Recorder (3DgazeR), have been successfully employed in several user studies (see Herman and Stachon, 2016; Herman et al, 2017; Herman et al., 2018a, Herman and Stachon, 2018).

\subsection{Design of 3DmoveR 2.0}

Certain weaknesses and drawbacks of the tools (3DmoveR 1.0, 3DtouchR and 3DgazeR) have been identified during their use, resulting in the need for modifications and improvements. Identified improvement requirements include:

- Preparation of stimuli: the preparation of stimuli for user testing (i.e. digital terrain models) was lengthy in the first version and largely had to be done manually.

- Recording the different types of interaction: there was a requirement to record the interaction using different control devices (PC mouse, keyboard, and touch screen), without the need to modify the tool further.

- Scalability of interaction settings: in the first version of the tool, it was not possible to modify the functionality of the keys or mouse buttons, for example, to swap the functionality of the left and right mouse buttons.

Other functional requirements, including those aimed at displaying instructions, storing user responses, opinions and obtaining objective information related to a user's performance, are similar to the first version of the tool. Non-functional requirements include the user-friendliness for the tool itself, its ability to be used on different platforms, the relationship between the application's performance and the resources it uses, and the tool's development testing process.

\subsection{Implementation of 3DmoveR 2.0}

3DmoveR 2.0 comprises a client and server side (see Fig. 1). The client side is built with HTML (HyperText Markup Language), JavaScript, jQuery, and Three.js. The recorded data 
from the client side are uploaded to a server side where they are stored through PHP scripts to Comma Separated Value (CSV) files (see Fig. 2).

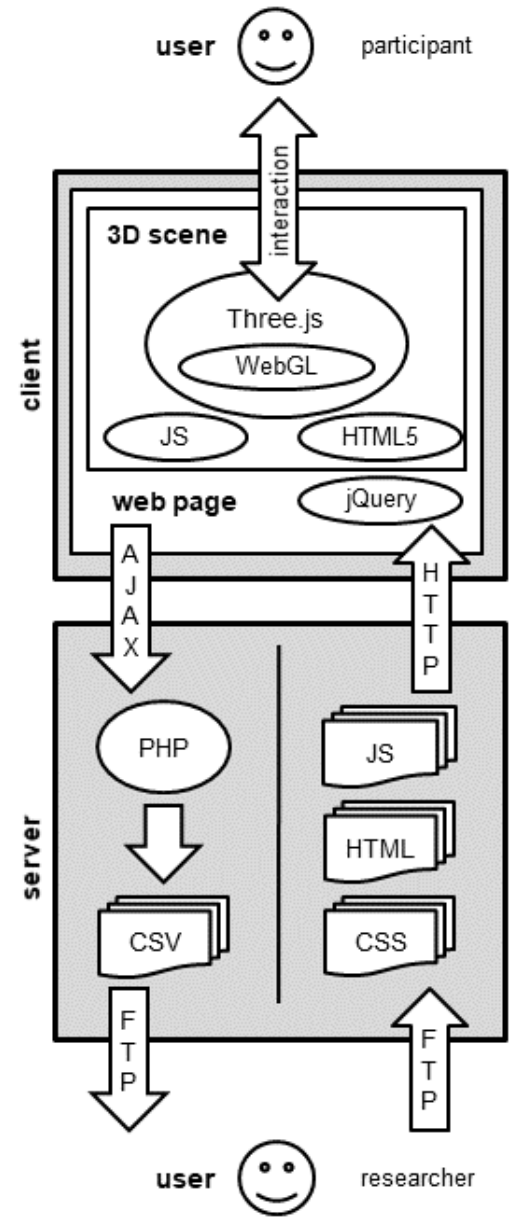

Fig. 1. The general architecture of the 3DmoveR 2.0.

From the technological point of view, the biggest change between version 1.0 and 2.0 was the replacement of the X3DOM library with Three.js. Three.js is a cross-browser JavaScript library and Application Programming Interface (API) used to create and display $3 \mathrm{D}$ graphics in a web browser. The first version was released by Ricardo Cabello in April 2010. Three.js uses WebGL (Web Graphics Library), and its source code is hosted in a repository on GitHub. Using Three.js extends the support for various types of devices (mousecontrolled desktop PCs and laptops with touchpads or tablets) and across all operating system platforms and web browsers.

In the Three.js facilitated implementation of the testing tool, the functions app.camera.position and app.controls.target are used to retrieve the position and orientation of the virtual camera. Input features such as buttons, checkboxes, radio buttons, and text boxes are implemented with conventional HTML. The captured movement and response data are stored in a JavaScript array on the client side and then posted on the server through Asynchronous JavaScript and XML (AJAX). PHP script creates CSV files on the server side, which are then downloaded by the researcher through File Transfer Protocol (FTP).

In addition to better hardware and software support of Three.js, this change had other benefits, such as automated and, therefore, faster stimuli preparation (using open source GIS-QGIS with the Qgis2threejs plugin), more precise stimuli control settings (assigning specific movements to different keys or prohibiting all types of movement for static stimuli), and the customisation of user movement in 3D scenes. This allowed for better control and greater accuracy than the previous 3 DmoveR version.

\begin{tabular}{|c|c|c|c|c|c|c|c|}
\hline \multicolumn{7}{|c|}{ Time $[\mathrm{ms}]$} & $\begin{array}{l}\text { Types of virtua } \\
\text { movement }\end{array}$ \\
\hline & \multicolumn{3}{|c|}{ Position $\left(X_{p}, Y_{p}, Z_{p}\right)$} & \multicolumn{3}{|c|}{ Orientation $\left(X_{0}, Y_{0}, Z_{0}\right)$} & \\
\hline & & & & & & & \\
\hline .. & $\cdots$ & $\cdots$ & $\cdots$ & $\cdots$ & $\cdots$ & $\cdots$ & $\cdots$ \\
\hline 5040 & -34.5471 & 36.58726 & 9.3223 & 7.821203 & -3.03181 & -2.15575 & ROTATE \\
\hline 5050 & -34.5471 & 36.58726 & 9.3223 & 7.821203 & -3.03181 & -2.15575 & WITHOUT \\
\hline 5060 & -34.9098 & 36.19582 & 9.3214 & 7.821203 & -3.03181 & -2.15575 & ROTATE \\
\hline 5090 & -35.0447 & 35.60246 & 9.3223 & 9.974087 & 0.88769 & 1.038079 & PAN \\
\hline 5100 & -36.2511 & 34.53505 & 9.796474 & 9.974087 & 0.88769 & 1.038079 & WITHOUT \\
\hline 5130 & -36.4234 & 34.33193 & 9.796474 & 9.974087 & 0.88769 & 1.038079 & ZOOM - WHEEL - IN \\
\hline$\ldots$ & $\ldots$ & $\ldots$ & $\ldots$ & $\ldots$ & $\ldots$ & $\ldots$ & $\ldots$ \\
\hline
\end{tabular}

Fig. 2. Example of data about virtual movement and user interaction.

Writing data to CSV files allows easy analysis using various open-source or freeware software (statistical: Open Office Calc, R; GIS: QGIS), as well as commercial software (statistical: MS Excel; GIS: ESRI ArcGIS, and FME).

\subsection{Evaluation}

Two methods were used to evaluate the 3DmoveR 2.0. To verify performance, capacity, and availability, we performed load testing through the JMeter application. In the second step, pilot user testing was carried out to verify the general accessibility and usability of the tool. The simple 3D scene shown in Fig. 3. was used in both steps.

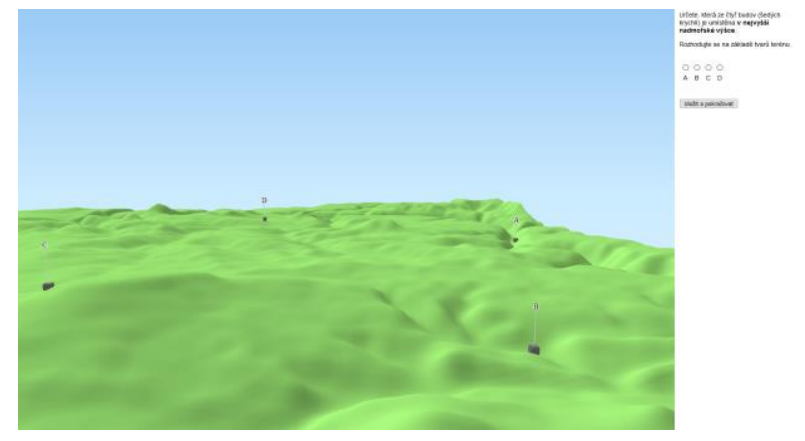

Fig. 3. Simple interactive 3D geovisualisation in 3DmoveR 2.0.

The results of the testing in JMeter are summarised in Fig. 4. In terms of availability $(90 \%)$, no problems have been identified as the application is not intended for high availability.

Pilot-testing users were approached via Facebook. 35 users attempted to fulfil the test, of which 30 continued through the whole testing process and completed the assignment. Of the participants, 17 were female and 18 male, aged between 21 and 56 years; four users did not give their ages. All participants reported that they worked daily with computers, and the majority $(94 \%)$ worked regularly with maps. However, there was a variation of answers regarding their experience with $3 \mathrm{D}$ (geo)visualisations, with some participants working with 3D daily and others reporting very low experience. 


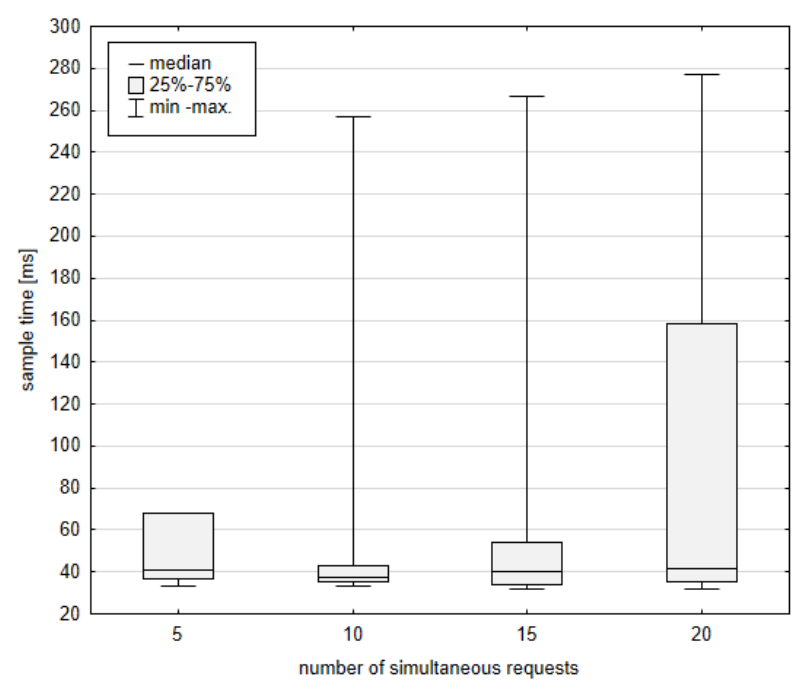

Fig. 4. Performance of 3DmoveR 2.0 at different loads.

Most users completed the test on personal computers or laptops; one participant used a tablet and four used smartphones. The participants used different operational systems: $74 \%$ worked on Windows, $11 \%$ on Android, $9 \%$ used variants of Linux, and the remaining $6 \%$ used macOS. They also used a variety of web browsers: $71 \%$ used Google Chrome, 14\% Mozilla Firefox, 9\% variants of Opera, and the remaining $6 \%$ other browsers, such as Safari or Internet Explorer

Of the vast majority of users who completed the test (30), only one complained about the loading speed of the 3D scene. One user reported troubles with controlling the app, and another reported problems with the layout of the geovisualisation on the screen, probably because he was not able to zoom out. This user was using a touchscreen device (Android 7.1; Opera mini). So 3DmoveR 2.0 may be considered sufficient even in terms of accessibility. We can also draw conclusions that for small mobile devices, better optimisation (so-called responsive design) would be needed.

Users answered the test question (choose object in highest altitude) in under one minute on average but with relatively wide variability $(\mathrm{m}=54.0 \mathrm{~s}$, $\mathrm{med}=43.7 \mathrm{~s}, \mathrm{stdv}=34.2 \mathrm{~s})$. Users were only partially correct when solving the task $(57 \%$ chose the correct object); $40 \%$ guessed the other object, which was placed almost as high as the correct object, as positioned in the highest altitude. With respect to the hardware/software platform participants used, no pattern was observed in terms of its effect on the speed or correctness of the responses.

The 3Dmove R application was able to implement all three main functional requirements mentioned in section 3.1. The stimuli can be easily prepared using QGIS with the Qgis2threejs plugin. The precise stimuli control settings and customisation of user movement in 3D scenes are also supported. In term of nonfunctional requirements, the results of our pilot tests showed that the application can be considered user friendly. Users did not report any major problems when using 3DmoveR 2.0. The tool's performance and capacity were also verified successfully. In terms of availability, no problems were identified.

\subsection{Application}

The first deployment of 3DmoveR 2.0 is described in detail by Herman et al. (2018b). This user study focuses on the influence of interactivity in virtual 3D geovisualsations on users' performance (the accuracy and speed of user responses). While some of the users were experienced in working with geospatial data, others were novices. The users completed a testing battery with various types of tasks, including both interactive and static 3D geovisualisations, under controlled conditions (in the laboratory). Google Chrome was used to launch the test, as the pilot study found that this web browser was the most commonly used.

The test battery comprised an introductory questionnaire on personal information and previous 3D (geo)visualisation experience followed by two training tasks (one static and one interactive) and 24 testing tasks. At the end of the test, the psychological Object-Spatial Imagery and Verbal Questionnaire (OSIVQ) was given (Herman et al., 2018b).

Significant differences in both accuracy and speed were found between static and interactive 3D geovisualisations. The collected data indicated that spatial tasks in 3D geovisualsations are solved better when interactivity is enabled and that users subjectively preferred to solve interactive tasks. On the other hand, tasks were solved faster with static geovisualisations. Differences between experts and novices in overall task solving accuracy were also found. Moreover, further analysis suggested that some differences may exist also between specific types of tasks (Herman et al., 2018b).

\section{DISCUSSION}

3DmoveR 2.0 works seamlessly in common web browsers and on both desktop and mobile devices. This allows us to use 3DmoveR 2.0 when testing outside controlled conditions (on user devices), which is important to obtain large samples of participants (users). Users would be able to perform the navigational task in a real environment (e.g. find a meeting point using interactive 3D city model on a mobile device) or to conduct an advanced spatial task, like analysing multicriterial analysis or "planning" (e.g. place a mobile signal transmitter, lookout tower, or hydroelectric power station in the optimal place on the virtual terrain).

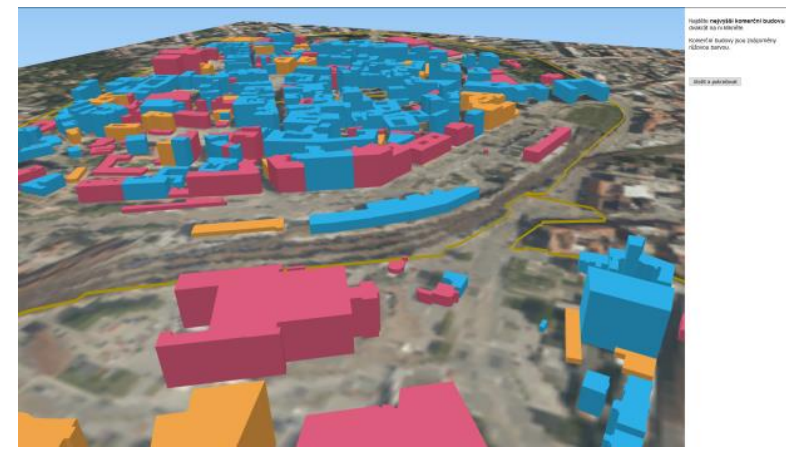

Fig. 5. Example of interactive 3D city model in 3DmoveR 2.0.

These complex tasks also require advanced types of interaction. However, the difficulty of these tasks is also affected by the shape and complexity of the terrain, the distance between the objects inserted into this terrain, and several other conditions that must or should be met. Therefore, it must also be mentioned that 3D geovisualisations represent relatively complex stimuli that do not allow a strict research design when preparing a user study. Hence, comprehensive data collection is required in interactive $3 \mathrm{D}$ geovisualisations to acquire better insight into the processes of decision-making and task-solving strategies. 


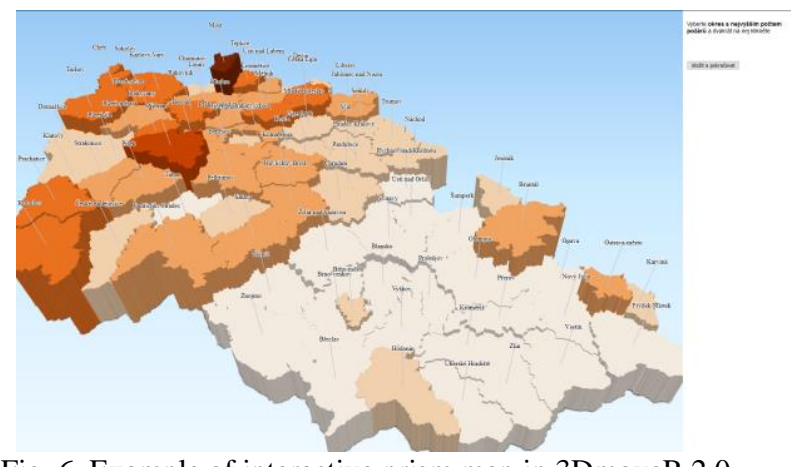

Fig. 6. Example of interactive prism map in 3DmoveR 2.0.

Although 3DmoveR 2.0 is primarily designed to test $3 \mathrm{D}$ geospatial data, it is also allows the creation of slides containing classic questionnaires (e.g. standardised psychological tests like the OSIVQ, Mental Rotation Test, or Object Visual Recognition Test). Psychological tests and their combination with the results of practical tasks performed using 3D geospatial data allow a better understanding of the inter-individual differences in user interaction and decision-making.

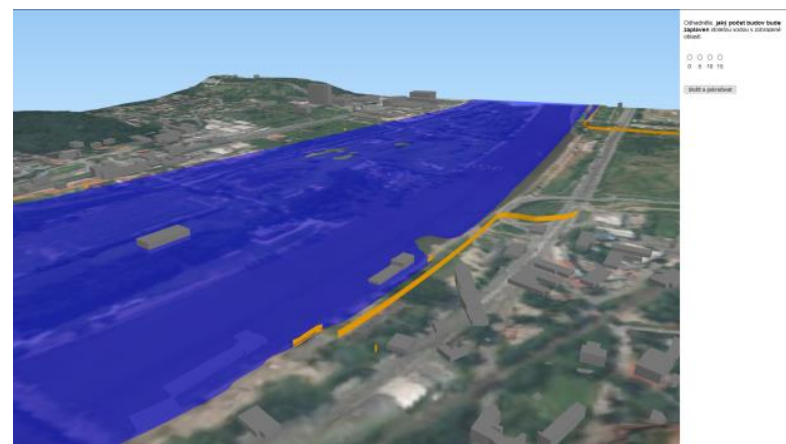

Fig. 7. Example of interactive 3D city model and flood map in 3DmoveR 2.0.

\section{CONCLUSIONS AND FUTURE WORK}

The 3DmoveR 2.0 software was successfully validated through a usability test involving interactive $3 \mathrm{D}$ geovisualisations. To summarise, the application has the following major advantages:

- It is based on freely available web technologies.

- It is freely available to any interested person operating under a Berkeley Software Distribution (BSD) license.

- Usability testing in 3DmoveR 2.0 does not require installing any special software.

- It is easily modifiable for different 3D scene contents (terrain, buildings, 3D symbols, textures, etc.), control positions, and many other variables (see Fig. 5, 6 and 7). It may also be modified for use in other fields or applications.

- It is versatile, recording data that can easily be used to calculate efficiency, effectiveness, and other aspects of usability or individual strategies. It collects both quantitative and qualitative data and can be combined with other usability research methods.

- Its recordings of user strategies offer researchers innovative ways to explore usability and other user aspects of interactive $3 \mathrm{D}$ geovisualisations.

Regarding the future development of 3 DmoveR, we want to focus on integration with other technologies, such as:

- $\quad$ specific JavaScript libraries (e.g. jsPsych and Webgazer.js),
- $\quad$ eye-tracking devices,

- WebVR and different head mounted displays (HMD), such as Google Cardboard or HTC Vive.

It would be also possible to use crowdsourcing as method of data collection through 3DmoveR 2.0. A 3D game (i.e. treasure hunt) seems the most promising way to crowdsource a 3D experiment. In this case, modification would be necessary to ensure more advanced data collection when testing outside a controlled environment. For this purpose, it is particularly necessary to develop procedures for better monitoring and control of environmental variables during user testing.

By presenting outcomes, knowledge about the usability and cognitive aspects of 3D geovisualisation can be expanded and explain indirectly at least some of the theoretical background of 3D geovisualisation. The use of testing tools, such as 3DmoveR 2.0, permits detailed user interaction analysis and is a benefit in this regard.

\section{ACKNOWLEDGEMENTS}

This paper is part of a project that has received funding from the European Union's Horizon 2020 research and innovation programme under grant agreement No 818346, titled "Sino-EU Soil Observatory for intelligent Land Use Management" (SIEUSOIL).

\section{REFERENCES}

Abend, P., et al., 2012. Geobrowsing Behaviour in Google Earth - A Semantic Video Content Analysis of On-Screen Navigation. In GI_Forum 2012: Geovisualization, Society and Learning, Jekel T. A. et al., Eds. pp. 2-13.

Biljecki, F., et al., 2015. Applications of 3D city models: State of the art review. ISPRS International Journal of GeoInformation, 4(4), 2842-2889. doi:10.3390/ijgi4042842.

Bleisch, S., 2011. Evaluating the Appropriateness of Visually Combining Quantitative Data Representations with 3D Desktop Virtual Environments Using Mixed Methods. PhD Dissertation, City University London, 2011. http://openaccess.city.ac.uk/1092/1/Bleisch\%2C_Susanne.pdf (15 October 2019).

Bleisch, S., et al., 2008. Evaluating the Effectiveness of Representing Numeric Information Through Abstract Graphics in 3D Desktop Virtual Environments. The Cartographic Journal 2008, 45(3), 216-226. doi:10.1515/10.1179/000870408X311404.

Cameron, R., 2009. A Sequential Mixed Model Research Design: Design, Analytical and Display Issues. International Journal of Multiple Research Approaches, 3(2), 140-152. doi:10.5172/mra.3.2.140.

Engel, J., et al., 2013. Evaluating the Perceptual Impact of Rendering Techniques on Thematic Color Mappings in 3D Virtual Environments. In Proceedings of 18th International Workshop on Vision, Modeling and Visualization (VMV 2013).

Herbert, G., Chen, X., 2015. A Comparison of Usefulness of 2D and 3D Representations of Urban Planning. Cartography and Geographic Information Science, 42(1), 22-32. doi:10.1080/15230406.2014.987694. 
Herman, L., et al., 2017. Eye-tracking Analysis of Interactive 3D Geovisualizations. Journal of Eye Movement Research, 10(3), 1-15. doi:10.16910/jemr.10.3.2.

Herman, L., et al., 2018a. Experimental Tool for Usability Testing of Interactive 3D Maps: Requirements, Design, Implementation, and Evaluation. Cartographic Perspectives, 2018(90), 31-63. doi:10.14714/CP90.1411.

Herman, L., et al., 2018b. Evaluation of User Performance in Interactive and Static 3D Maps. ISPRS International Journal of Geo-Information, 7(11), 1-25. doi:10.3390/ijgi7110415.

Herman, L., Stachon, Z., 2016. Comparison of User Performance with Interactive and Static 3D Visualization - Pilot Study. Int. Arch. Photogramm. Remote Sens. Spatial Inf. Sci., XLI-B2. pp. 655-661. doi:10.5194/isprs-archives-XLI-B2-6552016.

Herman, L., Stachon, Z., 2018. Controlling 3D Geovisualizations through Touch Screen - The Role of Users Age and Gesture Intuitiveness. In Proceedings, 7th International Conference on Cartography and GIS, Vol. 1. Sozopol, Bulgaria, Bandrova, T., Konecny, M., Eds.; Bulgarian Cartographic Association: Sofia, Bulgaria. pp. 473-480.

Jobst, M., Germanchis, T., 2007. The Employment of 3D in Cartography - An Overview. In Cartwright, W., Peterson, M. P., Gartner, G. (eds.) Multimedia Cartography, Springer: Berlin, Heidelberg, Germany, pp. 217-228.

Jurik, V., et al., 2017. When the Display Matters: A Multifaceted Perspective on 3D Geovisualizations. Open Geosciences, $9(1)$, 89-100. doi:10.1080/17538947.2017.1382581.

Li, X., et al., 2010. Visual Exploration of Eye Movement Data Using the Space-Time-Cube. In Fabrikant, S. I. (ed.) Geographic Information Science, Springer: Berlin, Heidelberg, Germany. pp. 295-309.

Liu, B., et al., 2017. Using Eye Tracking to Explore the Guidance and Constancy of Visual Variables in 3D Visualization. ISPRS International Journal of Geo-Information, 6(9), 1-18, doi:10.3390/ijgi6090274.

McKenzie, G., Klippel, A., 2016. The Interaction of Landmarks and Map Alignment in You-Are-Here Maps. The Cartographic Journal, 53(1), 43-54. doi:10.1179/1743277414Y.0000000101.

Niedomysl, T., et al., 2013. Learning Benefits of Using 2D versus 3D Maps: Evidence from a Randomized Controlled Experiment. Journal of Geography, 112(3), 87-96. doi:10.1080/00221341.2012.709876.

Nivala, A.-M., et al., 2008. Usability Evaluation of Web Mapping Sites. The Cartographic Journal, 45(2), 129-138. doi:10.1179/174327708X305120.

Popelka, S., Brychtova, A., 2013. Eye-tracking Study on Different Perception of 2D and 3D Terrain Visualization. The Cartographic Journal, 50(3), 240-375. doi:10.1179/1743277413Y.0000000058.

Preppernau, C. A., Jenny, B., 2015. Three-dimensional versus Conventional Volcanic Hazard Maps. Natural Hazards, 78(2), 1329-1347. doi:10.1007/s11069-015-1773-z.
Pucher, A., Schobesberger, D., 2011. Implicit User Logging as a Source for Enhancing the Usability of Web - Delivered Cartographic Applications. In Proceedings of ICC 2011 Paris. http://icaci.org/files/documents/ICC_proceedings/ICC2011/Oral \%20Presentations\%20PDF/B1-Fitness\%20for\%20use/CO067.pdf (15 October 2019).

Rautenbach, V., et al., 2016. Investigating the Use Of 3D Geovisualizations for Urban Design in Informal Settlement Upgrading in South Africa. Int. Arch. Photogramm. Remote Sens. Spatial Inf. Sci., XLI-B2. pp. 425-431. doi:10.5194/isprsarchives-XLI-B2-425-2016.

Reznik, T., 2013. Geographic information in the age of the INSPIRE Directive: discovery, download and use for geographical research. Geografie, 118(1), pp. 77-93.

Schobesberger, D., Patterson, T., 2007. Evaluating the Effectiveness of 2D vs. 3D Trailhead Maps. Mountain Mapping and Visualisation. In Proceedings of the 6th ICA Mountain Cartography Workshop Mountain Mapping and Visualisation. pp. 201-205.

Seipel, S., 2013. Evaluating 2D and 3D Geovisualisations for Basic Spatial Assessment. Behaviour \& Information Technology, 32(8), 845-858. doi:10.1080/0144929X.2012.661555.

Speicher, M., 2014. WaPPU: Usability-Based A/B Testing. In Web Engineering. ICWE 2014. Casteleyn, S., et al., Eds.; Springer: Cham, Germany. pp. 545-549. doi:10.1007/978-3319-08245-5_47.

Sprinarova, K.; et al., 2015. Human-computer Interaction in Real 3D and Pseudo-3D Cartographic Visualization: A Comparative Study. In Cartography - Maps Connecting the World: 27th International Cartographic Conference 2015 ICC2015. Sluter, C. R., et al., Eds.; Springer: Berlin, Germany. pp. $59-73$.

Treves, R., et al., 2015. Footprints in the Sky: Using Student Tracklogs from a "Bird's Eye View" Virtual Field Trip to Enhance Learning. Journal of Geography in Higher Education, 39(1), 97-110. doi:10.1080/03098265.2014.1003798.

Van Elzakker, C. P. J. M., 2004. The Use of Maps in the Exploration of Geographic Data. Netherlands Geographical Studies. PhD Dissertation, Universiteit Utrecht, 2004.

Van Elzakker, C. P. J. M., Griffin, A. 2013. Focus on Geoinformation Users. GIM International, 27(8), 20-23. Accessed October 15, 2019. https://www.giminternational.com/content/article/focus-on-geoinformation-users (15 October 2019).

Wilkening, J., Fabrikant, S. I., 2013. How Users Interact with a 3D Geo-Browser under Time Pressure. Cartography and Geographic Information Science, 40(1), 40-52. doi:10.1080/15230406.2013.762140.

Zhou, Y., et al., 2016. Enhanced 3D Visualization Techniques in Support of Indoor Location Planning. Computers, Environment and Urban Systems, 50, 15-29. doi:10.1016/j.compenvurbsys.2014.10.003. 